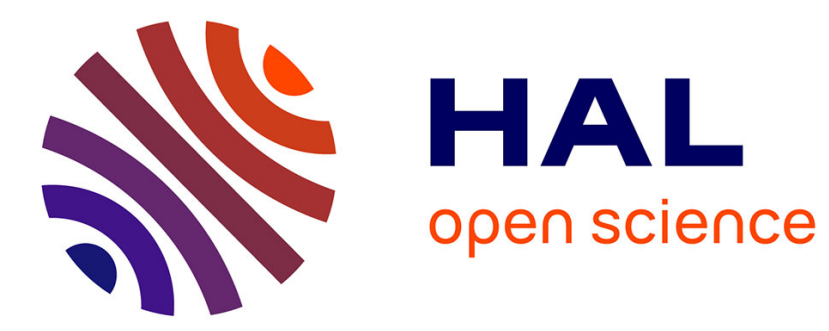

\title{
Model Reference Control for Timed Event Graphs in Dioids
}

Bertrand Cottenceau, Laurent Hardouin, Jean-Louis Boimond, Jean-Louis Ferrier

\section{- To cite this version:}

Bertrand Cottenceau, Laurent Hardouin, Jean-Louis Boimond, Jean-Louis Ferrier. Model Reference Control for Timed Event Graphs in Dioids. Automatica, 2001, 37, pp.1451-1458. hal-00845151

\section{HAL Id: hal-00845151 \\ https://hal.science/hal-00845151}

Submitted on 16 Jul 2013

HAL is a multi-disciplinary open access archive for the deposit and dissemination of scientific research documents, whether they are published or not. The documents may come from teaching and research institutions in France or abroad, or from public or private research centers.
L'archive ouverte pluridisciplinaire HAL, est destinée au dépôt et à la diffusion de documents scientifiques de niveau recherche, publiés ou non, émanant des établissements d'enseignement et de recherche français ou étrangers, des laboratoires publics ou privés. 


\title{
Model Reference Control for Timed Event Graphs in Dioids
}

\author{
B. Cottenceau, L. Hardouin, J.L. Boimond, J.L. Ferrier \\ Laboratoire d'Ingénierie des Systèmes Automatisés, \\ 62 av. Notre-Dame du Lac, 49000 ANGERS, FRANCE.
}

Tel: (33) 241365733

Fax: (33) 241365735.

\begin{abstract}
This paper deals with feedback controller synthesis for Timed Event Graphs in dioids. We discuss here the existence and the computation of a controller which leads to a closed-loop system whose behavior is as close as possible to the one of a given reference model and which delays as much as possible the input of tokens inside the (controlled) system. The synthesis presented here is mainly based on residuation theory results and some Kleene star properties.
\end{abstract}

Keywords: Discrete Event Systems, Timed Event Graphs, Dioid, Residuation Theory, Feedback Synthesis.

*bertrand.cottenceau@istia.univ-angers.fr 


\section{Introduction}

Timed Event Graphs (TEG) constitute a subclass of timed Petri nets of which each place has exactly one upstream and one downstream transition. It is well known that the timed/event behavior of a TEG, under the earliest functioning rule ${ }^{1}$, can be expressed by linear relations over some dioids (Baccelli et al., 1992) (De Schutter, 1996). Strong analogies then appear between the classical linear system theory and the $(\max ,+)$-linear system theory. In particular, the concept of control is well defined in the context of TEG study. It refers to the firing-control of the TEG input transitions in order to reach desired performance (see for instance (Cofer \& Garg, 1996) (Takai, 1989)). In the $(\max ,+)$ literature, an optimal control for TEG exists and is proposed in (Cohen et al., 1989)(Menguy et al., 2000). For a given reference input, this open-loop structure control yields the latest input firing date in order to obtain the output before the desired date.

This paper aims to transpose some closed-loop control structures to TEG. More precisely, we focus on controller synthesis such as output feedback controller, state feedback controller or output feedback on state controller. The controller synthesis is done in order that the controlled system will behave as close as possible to a given reference model. Furthermore the proposed controllers allow delaying as much as possible the token input inside the TEG.

Applications of these controllers are possible within the framework of production management. Indeed, TEG are well adapted to represent a class of manufacturing systems which present only delays and synchronization phenomena (Ayhan \& Wortman, 1999). Therefore, in the manufacturing context our controllers allow modifying the dynamics of a system (production line or manufacturing workshop) according to a given reference model and delaying as much as possible the raw parts input into the system. The latter property contributes to decreasing the work-in-process amount which is a permanent concern for the just-in-time production.

In the next section, we recall some theoretical results from the $(\max ,+)$ literature and introduce the algebraic foundations. Section 3 is devoted to recall some elements of TEG representation over particular dioids. The problem of controller synthesis is stated and solved in section 4 . Section 5 aims to present an illustrative example.

\section{Elements of Dioid and Residuation Theories}

\subsection{Dioid Theory}

We first recall in this section some notions from the dioid theory. The reader is invited to consult (Baccelli et al., 1992) for a complete presentation.

Definition 1 (Dioid) $A$ dioid is a set $\mathcal{D}$ endowed with two inner operations denoted $\oplus$ and $\otimes$. The sum is associative, commutative, idempotent $(\forall a \in \mathcal{D}, a \oplus a=a)$ and admits a neutral element denoted $\varepsilon$. The product is associative, distributes over the sum and admits a neutral element denoted $e$. The element $\varepsilon$ is absorbing for the product.

\footnotetext{
${ }^{1}$ i.e. a transition is fired as soon as it is enabled.
} 
Definition 2 (Order Relation) An order relation can be associated with a dioid $\mathcal{D}$ by the following equivalence: $\forall a, b \in \mathcal{D}, a \succeq b \Longleftrightarrow a=a \oplus b$.

Definition 3 (Complete Dioid) $A$ dioid $\mathcal{D}$ is complete if it is closed for infinite sums and if the product distributes over infinite sums too.

Example 1 ( $\overline{\mathbb{Z}}_{\text {max }}$ dioid) Set $\overline{\mathbb{Z}}=\mathbb{Z} \cup\{-\infty,+\infty\}$ endowed with the max operator as sum and the classical sum + as product is a complete dioid, usually denoted $\overline{\mathbb{Z}}_{\text {max }}$, of which $\varepsilon=-\infty$ and $e=0$.

The following theorem allows solving certain implicit equations defined over complete dioids.

Theorem 1 Over a complete dioid $\mathcal{D}$, the implicit equation $x=a x \oplus b$ admits $x=a^{*} b$ as least solution, where $a^{*}=\bigoplus_{i \in \mathbb{N}} a^{i}$ (Kleene star operator) with $a^{0}=e$.

Notation 1 The Kleene star operator, over a complete dioid $\mathcal{D}$, will be sometimes represented by the following mapping

$$
\begin{aligned}
\mathcal{K}: \mathcal{D} & \rightarrow \mathcal{D} \\
x & \mapsto \bigoplus_{i \in \mathbb{N}} x^{i} .
\end{aligned}
$$

The following theorem recalls some classical formulæ involving Kleene star mapping.

Property 1 Let $\mathcal{D}$ a complete dioid and $a, b \in \mathcal{D}$.

$$
\begin{aligned}
\left(a^{*}\right)^{*} & =a^{*} \\
a^{*} a^{*} & =a^{*} \\
a(b a)^{*} & =(a b)^{*} a .
\end{aligned}
$$

\subsection{Residuation Theory}

The residuation theory provides, under some assumptions, optimal solutions to inequalities such as $f(x) \preceq b$, where $f$ is an order-preserving mapping defined over ordered sets. Some theoretical results are recalled below. Complete presentations are given in (Blyth \& Janowitz, 1972) (Baccelli et al., 1992).

Definition 4 (Isotone mapping) A mapping $f$ defined over ordered sets is isotone if $a \preceq b \Rightarrow$ $f(a) \preceq f(b)$.

Definition 5 (Residual and residuated mapping) Let $f: \mathcal{E} \rightarrow \mathcal{F}$ an isotone mapping, where $(\mathcal{E}, \preceq)$ and $(\mathcal{F}, \preceq)$ are ordered sets. Mapping $f$ is said residuated if for all $y \in \mathcal{F}$, the least upper bound of subset $\{x \in \mathcal{E} \mid f(x) \preceq y\}$ exists and lies in this subset. It is then denoted $f^{\sharp}(y)$. Mapping $f^{\sharp}$ is called the residual of $f$. When $f$ is residuated, $f^{\sharp}$ is the unique isotone mapping such that

$$
f \circ f^{\sharp} \preceq \mathrm{Id} \text { and } f^{\sharp} \circ f \succeq \mathrm{Id},
$$

where Id is the identity mapping respectively on $\mathcal{F}$ and $\mathcal{E}$.

Theorem 2 ((Baccelli et al., 1992)) Let $f: E \rightarrow F$ where $E$ and $F$ are complete dioids of which bottom elements are respectively denoted $\varepsilon_{E}$ and $\varepsilon_{F}$. Then, $f$ is residuated iff $f\left(\varepsilon_{E}\right)=\varepsilon_{F}$ and $\forall A \subseteq E f\left(\bigoplus_{x \in A} x\right)=\bigoplus_{x \in A} f(x)$ 
Corollary 1 Mappings $x \mapsto$ ax and $x \mapsto x a$ defined over a complete dioid $\mathcal{D}$ are both residuated. Their residuals are usually denoted respectively $x \mapsto a \phi x$ and $x \mapsto x \phi a$ in (max, + ) literature.

proof: according to def.3, if $\mathcal{D}$ is a complete dioid then the product distributes over infinite sums and $\varepsilon$ is absorbing which satisfies the requirement of th. 2 .

Some classical results concerning product residual are given in the following theorem.

Theorem 3 ((Baccelli et al., 1992)) Mappings $x \mapsto a \phi x$ and $x \mapsto x \phi$ a verify the following properties:

$$
\begin{aligned}
& a \phi[a x] \succeq x \quad[x a] \phi a \succeq x \\
& a[a \phi a x]=a x \quad[x a \phi a] a=x a \\
& {[a b] \phi x=b \phi[a \phi x] \quad x \phi[b a]=[x \phi a] \phi b} \\
& {[a \phi x] b \preceq a \phi[x b] \quad b[x \phi a] \preceq[b x] \phi a} \\
& a^{*} x=a^{*} \phi\left[a^{*} x\right] \quad x a^{*}=\left[x a^{*}\right] \phi a^{*}
\end{aligned}
$$

Theorem 4 Let $\mathcal{D}$ a complete dioid and $A \in \mathcal{D}^{p \times n}$. Then, $A \oint A \in \mathcal{D}^{n \times n}$ and

$$
A ф A=(A \phi A)^{*} .
$$

proof: see (Max Plus, 1991) for another proof. First, according to (5), $A ф A \succeq e$, where $e \in \mathcal{D}^{n \times n}$ is the neutral element for matrix product. Moreover, according to (6), $A=A(A \phi A)$. Therefore, we have $A \phi A=A \phi[A(A \phi A)]$. Furthermore, thanks to (8), we can show that $A \phi[A(A \phi A)] \succeq$ $A ф A \otimes A ф A$. We thus obtain the following inequality $e \preceq(A \phi A)^{2} \preceq A \phi A$, and more generally $\forall n \in \mathbb{N}, e \preceq(A \phi A)^{n} \preceq A \phi A$. Therefore, we verify $e \preceq \bigoplus_{n \in \mathbb{N}}(A \phi A)^{n} \preceq A \phi A$ (i.e. $(A \phi A)^{*} \preceq A \phi A$ ) which finally leads to equality since, according to the dioid order definition (def.2) and the Kleene star definition (th.1), we also have $(A \phi A)^{*}=e \oplus A \phi A \oplus \cdots \succeq A \phi A$.

\subsection{Mapping restriction}

In this subsection, we address the problem of mapping restriction and its connection with the residuation theory. In particular, we show that the Kleene star mapping, which can be shown to be not residuated, becomes residuated as soon as its codomain is restricted to its image.

Definition 6 (Restricted mapping) Let $f: E \rightarrow F$ a mapping and $A \subseteq E$. We will denote $f_{\mid A}: A \rightarrow F$ the mapping defined by $f_{\mid A}=f \circ \operatorname{ld}_{\mid A}$ where $\operatorname{ld}_{\mid A}: A \rightarrow E, x \mapsto x$ is the canonical injection. Identically, let $B \subseteq F$ with $\operatorname{Im} f \subseteq B$. Mapping ${ }_{B \mid} f: E \rightarrow B$ is defined by $f=\operatorname{ld}_{\mid B}{ }^{\circ}{ }_{B \mid} f$, where $\operatorname{ld}_{\mid B}: B \rightarrow F, x \mapsto x$ is the canonical injection. 
Definition 7 (Closure mapping) An isotone mapping $f: E \rightarrow E$ defined on an ordered set $E$ is a closure mapping if $f \succeq \mathrm{Id}$ and $f \circ f=f$.

Remark 1 According to (1), mapping $\mathcal{K}$ is a closure mapping since $a^{*} \succeq$ a and $\left(a^{*}\right)^{*}=a^{*}$.

Proposition 1 Let a closure mapping $f: E \rightarrow E$. Then, $\left.\operatorname{Im} f\right|_{\mid} f$ is a residuated mapping whose residual is the canonical injection $\operatorname{ld}_{\mid \operatorname{Im} f}: \operatorname{Im} f \rightarrow E, x \mapsto x$.

proof: according to $(4),{ }_{\operatorname{Im} f \mid} f$ is residuated if there exists a mapping $g$ such that $\operatorname{Im} f \mid f \circ g \preceq \operatorname{Id}$ and $\left.g \circ \operatorname{Im} f\right|_{\mid} f \succeq \mathrm{Id}$, where identity mappings are respectively identity on $\operatorname{Im} f$ and on $E$. By setting $g=\operatorname{Id}_{\mid \operatorname{Im} f}$, we both verify $\left.\operatorname{Im} f\right|_{f} \circ \operatorname{Id}_{\mid \operatorname{Im} f}=\operatorname{Im} f \mid f_{\mid \operatorname{Im} f}=\operatorname{Id}$ (identity on $\operatorname{Im} f$ ) since $f \circ f=f$, and $\operatorname{Id}_{\mid \operatorname{Im} f} \circ \operatorname{Imf|} f=f \succeq \operatorname{ld}($ by def.7).

Corollary 2 Mapping $\operatorname{Im} \mathcal{K} \mid \mathcal{K}$ is a residuated mapping whose residual is $(\operatorname{Im} \mathcal{K} \mid \mathcal{K})^{\sharp}=\operatorname{Id}_{\mid \operatorname{Im} \mathcal{K}}$.

proof: the proof is direct since $\mathcal{K}$ is a closure mapping.

Remark 2 We can state from cor.2 that $x=a^{*}$ is the greatest solution to inequality $x^{*} \preceq a^{*}$. Actually, this greatest solution achieves equality.

\section{TEG description on dioids}

\subsection{Transfer function}

We recall that TEG can be seen as linear discrete event dynamical systems by using some dioid algebras (Cohen et al., 1989) (Baccelli et al., 1992). For instance, by associating with each transition

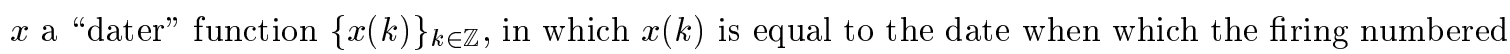
$k$ occurs, it is possible to obtain a linear state representation in $\overline{\mathbb{Z}}_{\text {max }}$. As in conventional system theory, output $\{y(k)\}_{k \in \mathbb{Z}}$ of a SISO TEG is then expressed as a convolution of its input $\{u(k)\}_{k \in \mathbb{Z}}$ by its impulse response ${ }^{2}\{h(k)\}_{k \in \mathbb{Z}}$.

Analogous transforms to $z$-transform (used to represent discrete-time trajectories in classical theory) can be introduced for TEG. Indeed, one can represent a dater $\{x(k)\}_{k \in \mathbb{Z}}$ by its $\gamma$-transform which is defined as the following formal power series: $X(\gamma)=\bigoplus_{k \in \mathbb{Z}} x(k) \gamma^{k}$. Variable $\gamma$ may also be regarded as the backward shift operator in event domain (formally, $\gamma x(k)=x(k-1)$ ). Consequently, one can express TEG behavior over the dioid of formal power series in one variable $\gamma$ and coefficients in $\overline{\mathbb{Z}}_{\text {max }}$. This dioid is usually denoted $\overline{\mathbb{Z}}_{\max } \llbracket \gamma \rrbracket$ in literature.

For instance, considering the TEG drawn in solid black lines in fig.2 (without taking account of the grey arcs), daters $x_{1}, x_{2}$ and $x_{3}$ are related as follows over $\overline{\mathbb{Z}}_{\text {max }}$ :

$$
x_{3}(k)=3 \otimes x_{1}(k-1) \oplus 8 \otimes x_{2}(k) \oplus 2 \otimes x_{3}(k-1) .
$$

\footnotetext{
${ }^{2}$ which is the ouput due to an infinity of input firings at date zero (Max Plus, 1991).
} 
Their respective $\gamma$-transforms, expressed over $\overline{\mathbb{Z}}_{\text {max }} \llbracket \gamma \rrbracket$, are then related as:

$$
x_{3}(\gamma)=3 \gamma x_{1}(\gamma) \oplus 8 x_{2}(\gamma) \oplus 2 \gamma x_{3}(\gamma)
$$

Consequently, for this TEG, we can obtain the following representation over $\overline{\mathbb{Z}}_{\text {max }} \llbracket \gamma \rrbracket$ :

$$
\left\{\begin{array}{l}
X=A X \oplus B U \\
Y=C X
\end{array}\right.
$$

with

$$
\begin{gathered}
A=\left(\begin{array}{ccc}
2 \gamma & \varepsilon & \varepsilon \\
\varepsilon & 3 \gamma & \varepsilon \\
3 \gamma & 8 & 2 \gamma
\end{array}\right), B=\left(\begin{array}{ll}
e & \varepsilon \\
\varepsilon & e \\
\varepsilon & \varepsilon
\end{array}\right), X=\left(\begin{array}{l}
x_{1} \\
x_{2} \\
x_{3}
\end{array}\right), \\
C=\left(\begin{array}{lll}
\varepsilon & \varepsilon & 2
\end{array}\right), U=\left(\begin{array}{l}
u_{1} \\
u_{2}
\end{array}\right) \text { and } Y=y .
\end{gathered}
$$

By solving the state equation of (11) according to th.1, i.e. $Y=C A^{*} B U$, we obtain the following transfer relation in $\overline{\mathbb{Z}}_{\text {max }} \llbracket \gamma \rrbracket$ :

$$
Y=\left(\begin{array}{ll}
5 \gamma(2 \gamma)^{*} & 10(3 \gamma)^{*}
\end{array}\right) U
$$

Remark 3 Algorithms and software tools are now available in order to establish such a transfer relation starting from the state representation (Gaubert, 1992) (Cottenceau, 1999).

\subsection{Periodicity, causality and realizability}

The transfer relation of a TEG is characterized by some periodic and causal properties that we recall hereafter. Let us consider a series $s=\bigoplus_{k \in \mathbb{Z}} s(k) \gamma^{k}$ in $\overline{\mathbb{Z}}_{\text {max }} \llbracket \gamma \rrbracket$. The support of $s$ is then defined by $\operatorname{Supp}(s)=\{k \in \mathbb{Z} \mid s(k) \neq \varepsilon\}$, and its valuation corresponds to the lower bound of $\operatorname{Supp}(s)$, i.e. $\operatorname{val}(s)=\min \{k \in \mathbb{Z} \mid s(k) \neq \varepsilon\}$. A series $s \in \overline{\mathbb{Z}}_{\text {max }} \llbracket \gamma \rrbracket$ such that $S u p p(s)$ is finite is said to be polynomial.

Definition 8 (Causality) A series $s \in \overline{\mathbb{Z}}_{m a x} \llbracket \gamma \rrbracket$ is causal if $s=\varepsilon$ or if $\{\operatorname{val}(s) \geq 0$ and $s \succeq$ $\left.\gamma^{\text {val(s) }}\right\}$. The set of causal elements of $\overline{\mathbb{Z}}_{\text {max }} \llbracket \gamma \rrbracket$ has a complete dioid structure denoted $\overline{\mathbb{Z}}_{\text {max }}^{+} \llbracket \gamma \rrbracket$.

Definition 9 (Periodicity) A series $s \in \overline{\mathbb{Z}}_{\text {max }} \llbracket \gamma \rrbracket$ is said to be periodic if it can be written as $s=p \oplus q\left(\tau \gamma^{\nu}\right)^{*}$ with $p$ and $q$ two polynomials and $\nu, \tau \in \mathbb{N}$. A matrix is said to be periodic if all its entries are periodic.

Definition 10 (Realizability) A series $s \in \overline{\mathbb{Z}}_{\max } \llbracket \gamma \rrbracket$ is said to be realizable if it exists three matrices $A, B$ and $C$ with entries in $\mathbb{N} \cup\{-\infty,+\infty\}$ such that $s=C(\gamma A)^{*} B$. A matrix is said to be realizable if all its entries are realizable.

In other words, a series $s$ is realizable if it corresponds to a transfer relation of a TEG.

Theorem 5 ((Cohen et al., 1989)) The following statements are equivalent:

- A series $s$ is realizable. 
- A series $s$ is periodic and causal.

The set of periodic series of $\overline{\mathbb{Z}}_{\max } \llbracket \gamma \rrbracket$ has a dioid structure which is not complete. Nevertheless, we have the following property.

Theorem 6 Let $s_{1}$ and $s_{2}$ two periodic series of $\overline{\mathbb{Z}}_{m a x} \llbracket \gamma \rrbracket$. Then, $s_{1} \phi s_{2}$ is also a periodic series. proof: see (Max Plus, 1991).

Theorem 7 The canonical injection $\mathrm{Id}_{\mid+}: \overline{\mathbb{Z}}_{\text {max }}^{+} \llbracket \gamma \rrbracket \rightarrow \overline{\mathbb{Z}}_{\text {max }} \llbracket \gamma \rrbracket$ is residuated. We denote $\operatorname{Pr}_{+}$: $\overline{\mathbb{Z}}_{\text {max }} \llbracket \gamma \rrbracket \rightarrow \overline{\mathbb{Z}}_{\text {max }}^{+} \llbracket \gamma \rrbracket$ its residual, i.e. $\operatorname{Pr}_{+}(s)$ is the greatest causal series less than or equal to $s$.

proof: see (Cottenceau et al., 1999).

From a practical point of view, for all $s \in \overline{\mathbb{Z}}_{\text {max }} \llbracket \gamma \rrbracket$, the computation of $\operatorname{Pr}_{+}(s)$ is obtained by:

$$
\operatorname{Pr}_{+}\left(\bigoplus_{k \in \mathbb{Z}} s(k) \gamma^{k}\right)=\bigoplus_{k \in \mathbb{Z}} s_{+}(k) \gamma^{k} \text { where } s_{+}(k)=\left\{\begin{array}{l}
s(k) \text { if }(k, s(k)) \geq(0,0) \\
\varepsilon \text { otherwise }
\end{array} .\right.
$$

Theorem 8 Let s a periodic (not necessary causal) series of $\overline{\mathbb{Z}}_{\text {max }} \llbracket \gamma \rrbracket$. Then, $\operatorname{Pr}_{+}(s)$ is the greatest realizable series less than or equal to $s$.

proof: (sketch of proof) the $\mathrm{Pr}_{+}$mapping simply amounts to zeroing terms of a series which are not with positive coefficient or exponent. Then, if $s$ is periodic, $\operatorname{Pr}_{+}(s)$ remains periodic. Finally, $\operatorname{Pr}_{+}(s)$ is both periodic and causal, i.e. realizable (cf. th.5).

\section{Feedback controller synthesis}

\subsection{Problem statement}

As presented previously, in dioid $\overline{\mathbb{Z}}_{\max } \llbracket \gamma \rrbracket$, the behavior of an $m$-inputs $p$-outputs TEG can be described by a state representation such as (11) where $U \in \overline{\mathbb{Z}}_{\text {max }} \llbracket \gamma \rrbracket^{m}$ and $Y \in \overline{\mathbb{Z}}_{\text {max }} \llbracket \gamma \rrbracket^{p}$. According to th.1, by solving the state equation in $X$, the input-output transfer relation is then expressed by

$$
Y=H U
$$

where $H=C A^{*} B$ belongs to $\overline{\mathbb{Z}}_{\text {max }} \llbracket \gamma \rrbracket^{p \times m}$.

We focus here on controller synthesis such as:

- output feedback controller: a controller, denoted $F$, is added between output $Y$ and input $U$ of the nominal system (see fig.1- $a$-). Therefore, the process input verifies $U=V \oplus F Y$, and the output is described by $Y=H(V \oplus F Y)$. According to th.1, the closed-loop transfer is then equal to

$$
Y=(H F)^{*} H V .
$$

- state feedback controller: a controller, denoted $L$, is added between internal $\operatorname{state}^{3} X$ and

${ }^{3}$ such a control structure implies that the internal state used for the control is measurable. 
input $U$ of the system (see fig.1-b-). The input is then described by $U=V \oplus L X$. First, by solving the state equation of (11) according to th.1, we have $X=A^{*} B U=A^{*} B(V \oplus L X)=$ $A^{*} B L X \oplus A^{*} B V$. Therefore, by solving this new implicit equation according to th.1, we obtain $X=\left(A^{*} B L\right)^{*} A^{*} B V$. Finally, by replacing $X$ in the output equation of (11) and by using (3) we have $Y=C\left(A^{*} B L\right)^{*} A^{*} B V=C A^{*} B\left(L A^{*} B\right)^{*} V$, which corresponds to the following transfer relation:

$$
Y=H\left(L A^{*} B\right)^{*} V .
$$

- output feedback on state controller: a controller, denoted $S$, is added between output and internal state (see fig.1-c-). The state evolution is then described by $X=A X \oplus B U \oplus S Y$. It is the reader's concern to check that the input-output transfer is given by

$$
Y=\left(C A^{*} S\right)^{*} H U \text {. }
$$

The controller $S$, located between output and internal state, behaves like inhibiting ${ }^{4}$ arcs. For instance, these arcs are depicted in grey lines in fig.2. Therefore, such a structure of control preserves a suitable meaning provided that one can effectively control the internal transitions, i.e. one can delay their firings when necessary.

The objective of the model reference control is to impose a desired behavior $\left(G_{r e f}\right)$ to a given system $(H)$ while finding the best controller carrying out this objective. More precisely, by denoting $G_{C}$ the transfer of the controlled system with controller $C$, we try to determine $C$ such that

$$
G_{C} \preceq G_{r e f} .
$$

Constraint (17) may be literally expressed as: the closed-loop system is at least as fast as the reference model.

In addition, by assuming that it may exist several controllers $C_{i}, i \in\{1, \ldots, n\}$, leading to the same controlled transfer, i.e. $G_{C_{0}}=\cdots=G_{C_{n}}$, we focus on the greatest one (when such an optimal exists): the greatest is the one which delays as much as possible the input in the system. Therefore, in the TEG context, this supremal controller minimizes the amount of tokens in the controlled TEG.

In short, for a given reference model, the problem tackled here consists in finding the greatest controller $C$ (when it exists) checking $G_{C} \preceq G_{r e f}$. Therefore, within the framework of feedback synthesis and according to (14)-(16), we have to find, for a given $G_{r e f}$, a greatest solution in $F$ (resp. $L$ and $S$ ) for inequality (18) (resp. (19) and (20))

$$
\begin{aligned}
(H F)^{*} H & \preceq G_{r e f} \\
H\left(L A^{*} B\right)^{*} & \preceq G_{r e f} \\
\left(C A^{*} S\right)^{*} H & \preceq G_{r e f} .
\end{aligned}
$$

In other words, this amounts to being interested in the properties of mappings $x \mapsto(H x)^{*} H$, $x \mapsto H\left(x A^{*} B\right)^{*}$ and $x \mapsto\left(C A^{*} x\right)^{*} H$ with respect to the residuation theory.

\footnotetext{
${ }^{4}$ the supplementary arcs due to the controller authorize or prohibit the firing of the controlled transitions.
} 


\subsection{Ouput feedback synthesis}

Let us define

$$
\begin{aligned}
M_{H}: \overline{\mathbb{Z}}_{\text {max }} \llbracket \gamma \rrbracket^{m \times p} & \rightarrow \overline{\mathbb{Z}}_{\max } \llbracket \gamma \rrbracket^{p \times m} \\
X & \mapsto(H X)^{*} H .
\end{aligned}
$$

This mapping clearly represents how an output feedback $X$ influences the closed-loop transfer dynamics. Clearly, inequality (18) admits a greatest solution for all reference models $G_{r e f}$ only if $M_{H}$ is residuated. However, according to th.2, one easily checks that $M_{H}$ is not residuated since $M_{H}(\varepsilon)=H \neq \varepsilon$. Nevertheless, the following result shows that there are restrictions of mapping $M_{H}$ which are residuated. That amounts to saying that the inequality (18) admits an optimal solution only for specific right-hand sides.

Proposition 2 Let $G \in \overline{\mathbb{Z}}_{\max } \llbracket \gamma \rrbracket^{p \times m}$ and $D \in \overline{\mathbb{Z}}_{\text {max }} \llbracket \gamma \rrbracket^{p \times p}$. Let us consider the following sets:

$$
\begin{aligned}
& \mathcal{G}_{1}=\left\{G \mid \exists D \text { periodic and causal s.t. } G=D^{*} H\right\} \\
& \mathcal{G}_{2}=\left\{G \mid \exists D \text { periodic and causal s.t. } G=H D^{*}\right\} .
\end{aligned}
$$

Mappings $\mathcal{G}_{1} \mid M_{H}$ and $\mathcal{G}_{2} \mid M_{H}$ are both residuated. Their residuals are such that $\left(\mathcal{G}_{1} \mid M_{H}\right)^{\sharp}(x)=$ $\left(\mathcal{G}_{2} \mid M_{H}\right)^{\sharp}(x)=H \phi x \phi H$.

proof: according to def.5, we remark that the two following assertions are equivalent:

- $\mathcal{G}_{1} \mid M_{H}$ is residuated

- $\forall D$ periodic and causal, $(H X) H^{*} \preceq D^{*} H$ admits a greatest solution.

So, we can concentrate on the second point. Since mapping $x \mapsto H x$ is residuated (cf. cor.1) and according to (3), we have:

$$
(H X)^{*} H=H(X H)^{*} \preceq D^{*} H \Longleftrightarrow(X H)^{*} \preceq H \phi\left(D^{*} H\right) .
$$

According to (9) and (7), we can rewrite

$$
H \phi\left(D^{*} H\right)=H \phi\left[D^{*} \phi\left(D^{*} H\right)\right]=\left(D^{*} H\right) \phi\left(D^{*} H\right) .
$$

According to (10), this last expression shows that $H \phi\left(D^{*} H\right)$ belongs to the image of $\mathcal{K}: \overline{\mathbb{Z}}_{\text {max }} \llbracket \gamma \rrbracket^{m \times m} \rightarrow$ $\overline{\mathbb{Z}}_{\text {max }} \llbracket \gamma \rrbracket^{m \times m}$. Since $\left.\operatorname{Im} \mathcal{K}\right|_{\mathcal{K}}$ is residuated (cf cor.2), there is also the following equivalence:

$$
(X H)^{*} \preceq H \phi\left(D^{*} H\right) \Longleftrightarrow X H \preceq H \phi\left(D^{*} H\right) .
$$

Finally, since mapping $x \mapsto x H$ is residuated too (cf. cor.1), we verify that $X=H \phi\left(D^{*} H\right) \phi H$ is the greatest solution of $H(X H)^{*} \preceq D^{*} H, \forall D \in \overline{\mathbb{Z}}_{m a x} \llbracket \gamma \rrbracket^{p \times p}$. That amounts to saying that $\mathcal{G}_{1} M_{H}$ is residuated. We would show that $\mathcal{G}_{2} \mid M_{H}$ is residuated with analog steps.

As recalled in section 3, working on TEG comes down to considering only the subset of periodic and causal series of $\overline{\mathbb{Z}}_{\max } \llbracket \gamma \rrbracket$ (cf. th.5). Then, the results obtained in prop.2 must be restricted to that case in order to be applied to TEG control. 
Proposition 3 If $G_{\text {ref }} \in \mathcal{G}_{1} \cup \mathcal{G}_{2}$, there exists a greatest realizable output feedback $F_{\mathrm{r}}$ such that $\left(H F_{\mathrm{r}}\right)^{*} H \preceq G_{r e f}$. This greatest controller is given by

$$
F_{\mathrm{r}}=\operatorname{Pr}_{+}\left(H \phi G_{r e f} \phi H\right) .
$$

proof: according to prop.2, $H \oint G_{r e f} \phi H$ is the greatest solution to $(H X)^{*} H \preceq G_{r e f}$. Since $G_{r e f} \in$ $\mathcal{G}_{1} \cup \mathcal{G}_{2}, G_{r e f}$ is periodic and causal. Therefore, according to th.6, $H \phi G_{r e f} \phi H$ is periodic. Eventually, according to th.8, $\operatorname{Pr}_{+}\left(H \phi G_{r e f} \phi H\right)$ is the greatest realizable solution.

\subsection{State feedback, feedback between output and state.}

For these two feedback synthesis problems, it is still a question of checking whether mappings $x \mapsto H\left(x A^{*} B\right)^{*}$ and $x \mapsto\left(C A^{*} x\right)^{*} H$ are residuated or not. According to th.2, it is clear that they are not residuated. Nevertheless, the problem of reference model control may have an optimal realizable solution, in each case, if $G_{r e f}$ is constrained to belong to particular subsets of $\overline{\mathbb{Z}}_{\text {max }} \llbracket \gamma \rrbracket^{p \times m}$.

Proposition 4 Let $H=C A^{*} B$ be a TEG transfer matrix. For all reference model $G_{r e f} \in \mathcal{G}_{1}$, there exists a greatest realizable state feedback $L_{\mathrm{r}}$ such that $H\left(L_{\mathrm{r}} A^{*} B\right)^{*} \preceq G_{r e f}$. This optimal solution is then computed by

$$
L_{\mathbf{r}}=\operatorname{Pr}_{+}\left(H \phi G_{r e f} \phi\left(A^{*} B\right)\right) .
$$

proof: as in the prop.2 proof, we first have to show that for all $D \in \overline{\mathbb{Z}}_{\text {max }} \llbracket \gamma \rrbracket^{p \times p}$, equation $H\left(L A^{*} B\right)^{*} \preceq D^{*} H$ admits a greatest solution. Since mapping $x \mapsto H x$ is residuated, we have:

$$
H\left(L A^{*} B\right)^{*} \preceq D^{*} H \Longleftrightarrow\left(L A^{*} B\right)^{*} \preceq H \phi\left(D^{*} H\right) .
$$

Moreover, we have shown in the prop.2 proof that element $H \phi\left(D^{*} H\right)$ belongs to the image of $\mathcal{K}: \overline{\mathbb{Z}}_{\text {max }} \llbracket \gamma \rrbracket^{m \times m} \rightarrow \overline{\mathbb{Z}}_{\text {max }} \llbracket \gamma \rrbracket^{m \times m}$. Then, since ${ }_{\operatorname{Im} \mathcal{K} \mid} \mathcal{K}$ is residuated,

$$
\left(L A^{*} B\right)^{*} \preceq H \phi\left(D^{*} H\right) \Longleftrightarrow L A^{*} B \preceq H \phi\left(D^{*} H\right) .
$$

Since $x \mapsto x H$ is residuated too, we then obtain that $H \oint\left(D^{*} H\right) \phi\left(A^{*} B\right)$ is the greatest solution to $H\left(L A^{*} B\right)^{*} \preceq D^{*} H$. Finally, if $G_{r e f}$ belongs to $\mathcal{G}_{1}, H \nmid G_{r e f} \phi\left(A^{*} B\right)$ is a periodic matrix (by applying th.6), and $\operatorname{Pr}_{+}\left(H \nmid G_{r e f} \phi\left(A^{*} B\right)\right)$ is then the greatest realizable solution (by applying th.8).

Proposition 5 Let $H=C A^{*} B$ be a TEG transfer matrix. For all reference model $G_{r e f} \in \mathcal{G}_{2}$, there exists a greatest realizable output feedback on state $S_{\mathrm{r}}$ such that $\left(C A^{*} S_{\mathrm{r}}\right)^{*} H \preceq G_{r e f}$. This optimal solution is then computed by

$$
S_{\mathrm{r}}=\operatorname{Pr}_{+}\left(\left(C A^{*}\right) \phi G_{r e f} \phi H\right) .
$$

proof: similar to the previous proof. 
Remark 4 (Particular case $\left.G_{r e f}=H.\right)$ Since the identity matrix e is such that $e^{*}=e$, we can easily check that $H \in \mathcal{G}_{1}$ and $H \in \mathcal{G}_{2}$. Therefore, for any TEG, it is possible to preserve its own transfer with either a greatest realizable output feedback, a greatest realizable state feedback or a greatest realizable output feedback on state. In (Cottenceau et al., 1999), that particular case has already been studied for output feedback control.

\section{Example}

In order to illustrate results presented previously, we describe a complete synthesis of an output feedback on state for the TEG depicted with solid black lines in fig.2. We assume that this model represents a workshop with 3 machines $\left(M_{1}, M_{2}, M_{3}\right)$ of which inputs are described by transitions $x_{1}, x_{2}$ and $x_{3}$. Therefore, this example corresponds to a short application of our results in the domain of manufacturing management.

We propose to compute a greatest output feedback on state so that the system has a transfer relation close to a given reference transfer $G_{r e f}$. For this TEG, according to section 3 , we have

$$
H=\left(5 \gamma(2 \gamma)^{*} \quad 10(3 \gamma)^{*}\right)
$$

This transfer shows the difference that exists between the production rate of path $u_{1} \rightarrow y$, namely $1 / 2$ token/time unit, and those of path $u_{2} \rightarrow y$, namely $1 / 3$ token/time unit.

According to the structure of the workshop, an unstability ${ }^{5}$ problem arises as soon as too many parts are admitted at the same time at inputs $u_{1}$ and $u_{2}$ because of the difference of production rates of machines $M_{1}$ and $M_{2}$. Indeed, in such a case, the marking of the place located between $x_{1}$ and $x_{3}$ will grow without bound. So, a realistic objective would be here to impose, thanks to the controller, the production rate of the slowest machine $\left(M_{2}\right)$ to the whole system (i.e. 1 part per 3 time units). According to prop.5, the model reference control has an optimal solution if $G_{r e f}$ both belongs to $\mathcal{G}_{2}$ and reflects the desired production rate. For instance, if we choose here $G_{r e f}=H(3 \gamma)^{*}$, we obtain

$$
G_{r e f}=\left(\begin{array}{ll}
5 \gamma(3 \gamma)^{*} & \left.10(3 \gamma)^{*}\right)
\end{array}\right.
$$

which satisfies both constraints.

Then, according to prop.5, the greatest realizable feedback $S_{\mathrm{r}}$ is given by computing $\operatorname{Pr}_{+}\left(\left(C A^{*}\right) \phi G_{r e f} \phi H\right)$. According to state representation (11), we have

$$
C A^{*}=\left(\begin{array}{lll}
5 \gamma(2 \gamma)^{*} & 10(3 \gamma)^{*} \quad 2(2 \gamma)^{*}
\end{array}\right)
$$

Therefore, we can compute the controller. First, we obtain

$$
\left(C A^{*}\right) \phi G_{r e f} \phi H=\left(\begin{array}{c}
-5 \gamma^{-1}(3 \gamma)^{*} \\
-10(3 \gamma)^{*} \\
-2(3 \gamma)^{*}
\end{array}\right)
$$

\footnotetext{
${ }^{5} \mathrm{a}$ TEG is said stable if the marking of all its internal places remains bounded for all input sequence (Max Plus, 1991). The problem of TEG stabilization has recently been reconsidered in (Commault, 1998).
} 
which is clearly not causal (but periodic) and then

$$
S_{\mathrm{r}}=\operatorname{Pr}_{+}\left(\left(C A^{*}\right) \phi G_{r e f} \phi H\right)=\left(\begin{array}{c}
1 \gamma(3 \gamma)^{*} \\
2 \gamma^{4}(3 \gamma)^{*} \\
1 \gamma(3 \gamma)^{*}
\end{array}\right)
$$

A realization of that optimal controller is drawn in grey lines in fig.2.

Remark 5 Some other examples are developed in (Cottenceau et al., 1999) (output feedback), (Cohen et al., 1998) (output feedback) and (Cottenceau, 1999) (all these structures are illustrated). Let us note that such a synthesis is indifferently obtained in either dioid $\overline{\mathbb{Z}}_{\text {max }} \llbracket \gamma \rrbracket$ or dioids $\overline{\mathbb{Z}}_{\text {min }} \llbracket \delta \rrbracket$ and $\mathcal{M}_{\text {in }}^{a x} \llbracket \gamma, \delta \rrbracket$. 


\section{References}

Ayhan, H. \& Wortman, M. (1999). Job flow control in assembly operations. IEEE TAC, 44(4), $864-868$.

Baccelli, F., Cohen, G., Olsder, G., \& Quadrat, J. (1992). Synchronization and Linearity: An Algebra for Discrete Event Systems. New York: John Wiley and Sons.

Blyth, T. \& Janowitz, M. (1972). Residuation Theory. Oxford: Pergamon Press.

Cofer, D. D. \& Garg, V. K. (1996). Supervisory control of real-time discrete-event systems using lattice theory. IEEE TAC, 41(2), 199-209.

Cohen, G., Gaubert, S., \& Quadrat, J. (1998). Max-plus algebra and system theory : Where we are and where to go now. In IFAC Conference on System Structure and Control Nantes.

Cohen, G., Moller, P., Quadrat, J., \& Viot, M. (1989). Algebraic Tools for the Performance Evaluation of Discrete Event Systems. IEEE Proceedings: Special issue on Discrete Event Systems, $77(1), 39-58$.

Commault, C. (1998). Feedback stabilization of some event graph models. IEEE Trans. on Automatic Control, 43(10), 1419-1423.

Cottenceau, B. (1999). Contribution à la commande de systèmes à événements discrets : synthèse de correcteurs pour les graphes d'événements temporisés dans les dioïdes. Ph.d. thesis (in french), ISTIA Université d'Angers.

Cottenceau, B., Hardouin, L., Boimond, J., \& Ferrier, J. (1999). Synthesis of greatest linear feedback for timed event graphs in dioid. IEEE Trans. on Automatic Control, 44(6), 1258-1262.

De Schutter, B. (1996). Max-algebraic system theory for discrete event systems. Ph. d. thesis, KU Leuven.

Gaubert, S. (1992). Théorie des systèmes linéaires dans les dioïdes. Ph.d. thesis (in french), Ecole des Mines de Paris, Paris.

Max Plus (1991). Second Order Theory of Min-linear Systems and its Application to Discrete Event Systems. In Proceedings of the 30th CDC Brighton, England.

Menguy, E., Boimond, J., Hardouin, L., \& Ferrier, J. (2000). Just in time control of timed event graphs : Update of reference input, presence of uncontrollable input. IEEE Trans. on Aut. Cont. (to appear).

Takai, S. (1989). A characterization of realizable behavior in supervisory control of timed event graphs. IEEE TAC, 77, 81-98. 


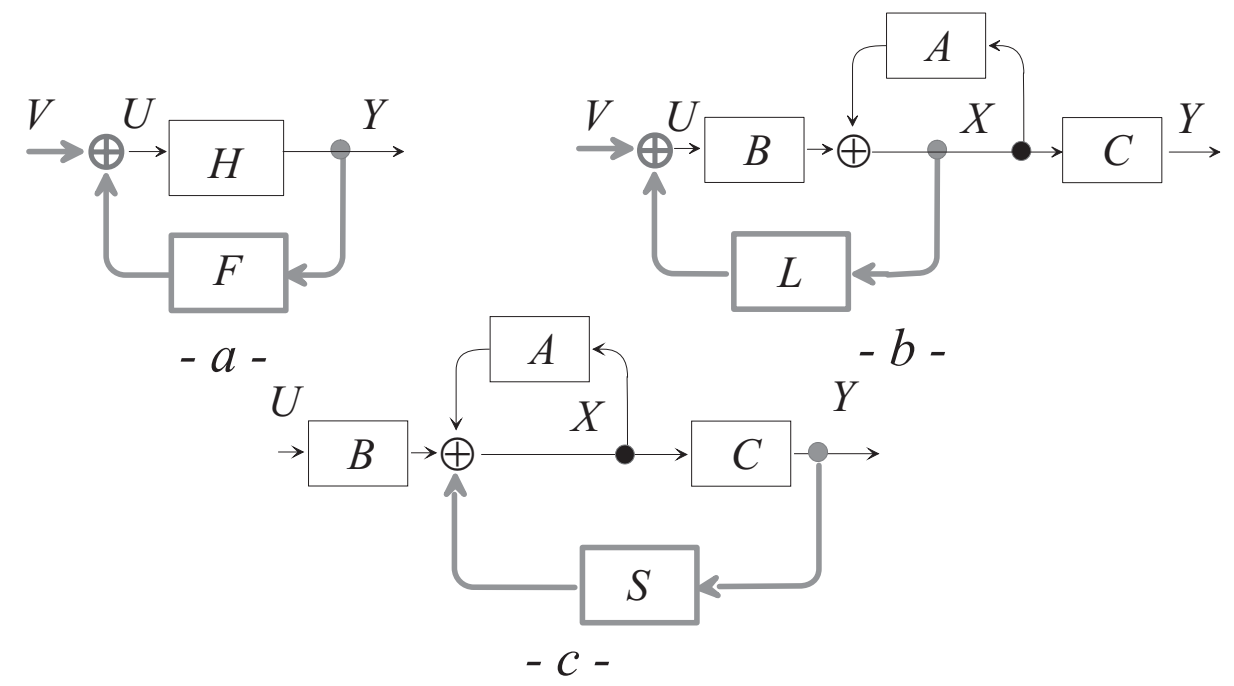

Figure 1: System $H=C A^{*} B$ with an output feedback $F(-a-)$, a state feedback $L(-b-)$ and an output feedback on state $S(-c-)$.

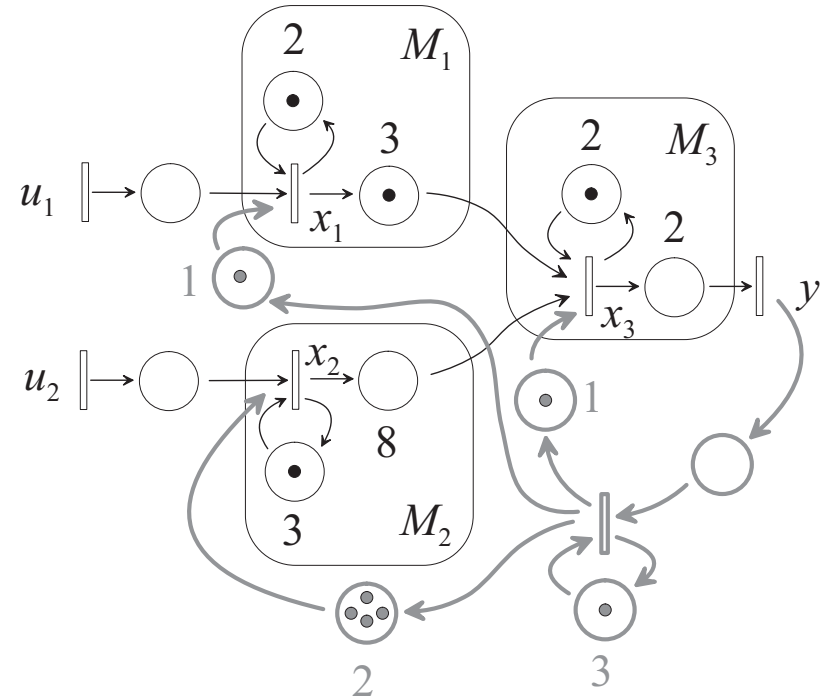

Figure 2: A TEG endowed with a controller (grey lines). 